\title{
Dynamic electrophoresis of a spherical dispersion of soft particles subject to a stress-jump condition
}

\author{
Wei-Lun Min, Eric Lee, Jyh-Ping Hsu* \\ Department of Chemical Engineering, National Taiwan University, Taipei 10617, Taiwan \\ Received 17 December 2005; accepted 18 January 2006 \\ Available online 9 March 2006
}

\begin{abstract}
The dynamic mobility of a spherical dispersion of soft particles, where a particle comprises a rigid core and a membrane layer, is evaluated for the case when the shear stress across the membrane layer-liquid interface is discontinuous, the so-called stress-jump condition. We show that, due to the effect of double-layer deformation, the magnitude of the dynamic mobility of a particle has a local maximum and the corresponding phase angle has a negative (phase lead) local minimum at a low to medium level of the frequency of the applied electric field. This effect becomes insignificant if the frequency of the applied electric field is sufficiently high. The stress-jump condition may lead to a significant influence on the drag, and consequently the mobility of a particle. The degree of influence depends upon the sign of the stress-jump coefficient and the charged conditions of the membrane layer of the particle.
\end{abstract}

(c) 2006 Elsevier Inc. All rights reserved.

Keywords: Dynamic electrophoresis; Spherical dispersion; Soft particles; Stress-jump condition

\section{Introduction}

Owing to their potential applications, such as the estimation of the surface potential of a colloidal entity in a concentrated dispersion, electroacoustic phenomena have drawn the attention of colloidal scientists in recent years. These phenomena include two reversal effects: applying a supersonic wave to a colloidal dispersion and measuring the responding vibration potential, and applying an alternating electric field and detecting the responding supersonic wave [1]. Several theoretical attempts have been made in the past decade to model electroacoustic phenomena [2-10]. The key factors considered in these studies include the level of the surface potential of a particle, the thickness of the double layer surrounding a particle, the concentration of particles, and the, types of boundary conditions assumed. Compared with the response of a colloidal dispersion to a static applied electric field, the response of a dynamic applied electric field is much more complicated. For instance, the electrophoretic velocity of a particle may not synchronize with the

\footnotetext{
* Corresponding author. Fax: +886223623040.

E-mail address: jphsu@ntu.edu.tw (J.-P. Hsu).
}

applied electric field; both phase lag and phase lead may be observed. Also, depending upon the operating conditions, both the magnitude and the phase angle of the electrophoretic velocity of a particle can have a complicated relation with the frequency of the applied electric field.

Previous analyses of electroacoustic phenomena all focused on dispersions of rigid entities. While particles of a rigid nature are common, those of nonrigid nature are also of practical significance. Biocolloids such as biological cells and microorganisms, for instance, belong to this category, in that their surfaces can be penetrable to ionic species. A so-called soft particle [11-14], where a particle comprises a rigid core and an ion-penetrable membrane layer, is often adopted to simulate these types of particles. Available results for the behavior of a soft particle when an alternating electric field is applied are very limited. Under conditions of low particle concentration, thick membrane layer, and thin double layer, Ohshima [15] was able to derive an analytical expression for the dynamic electrophoretic mobility of a soft particle. Lopez-Garcia et al. [16] proposed a numerical procedure to solve the problem of Ohshima; an extra hydrodynamic force acting on the rigid core of a particle was considered. In the mathematical treatment of these analyses, it was assumed that both the fluid velocity 
and its shear stress are continuous across the membrane layerliquid interface. In a study of the flow field involving a porous medium, Ochoa-Tapia and Whitaker $[17,18]$ were able to show that while the former is appropriate, the latter needs modification. A stress-jump condition, characterized by a stress-jump coefficient, was proposed to describe the shear stress across the porous medium-liquid interface. Their treatment was found to be more convincing than the empirical relation proposed by Beavers and Joseph [19]. Follow-up studies [20-22] also revealed that the introduction of a stress-jump condition for the description of the nature of the shear stress across a porous medium-liquid interface is necessary. Sekhar and Sano [21] suggested that the stress-jump coefficient ranges approximately from -0.9 to 0.9 .

In this study the electrophoresis of a spherical dispersion of soft particles, where a particle comprises a rigid core and an ion-penetrable membrane layer, subject to an alternating electric field is analyzed. In particular, the influence of the discontinuity in the shear stress across the membrane layer-liquid interface on the electrophoretic behavior of a dispersion is investigated. The dynamic electrophoretic mobility of a particle characterized by its magnitude and phase angle is evaluated under various conditions.

\section{Theory}

Let us consider the dynamic electrophoresis of a spherical dispersion of soft particles, where a particle comprises a rigid core of radius $a$ and a membrane layer of thickness $d$. The dispersion medium contains $z_{1}: z_{2}$ electrolyte; $z_{1}$ and $z_{2}$ are respectively the valence of cations and that of anions with $z_{2}=-\alpha z_{1}$. Referring to Fig. 1, the unit cell model of Kuwabara [23] is adopted, where the dispersion is simulated by a representative particle and a concentric spherical liquid shell of radius $c$. Let $b=a+d$ and $H=(b / c)^{3}$; the latter is a measure for the volume fraction of particles. The spherical coordinates $(r, \theta, \varphi)$ are adopted with origin located at the center of the particle. $\mathbf{E}=E_{Z} e^{-i \omega t} \mathbf{e}_{z}$ is the applied electric field, where $\mathbf{e}_{z}$ is the unit vector in the $z$-direction, $t$ is the time, $\omega$ is the frequency of $\mathbf{E}$, and $i=\sqrt{-1} . \mathbf{U}=U e^{-i \omega t} \mathbf{e}_{z}=U_{\mathrm{R}}+i U_{\mathrm{I}}$ is the electrophoretic velocity of the particle; $U_{\mathrm{R}}$ and $U_{\mathrm{I}}$ are respectively the real and the imaginary parts of $\mathbf{U}$. For the present case the electric potential $\phi$ can be described by the Poisson equation

$\nabla^{2} \phi=-\frac{\rho+\ell \rho_{\mathrm{fix}}}{\varepsilon}$,

where $\rho=\sum_{j} n_{j} z_{j} \hat{e}$ and $\rho_{\mathrm{fix}}$ are respectively the space density of mobile ions and the fixed-charge density in the membrane layer, $\nabla$ is the gradient operator, $\hat{e}$ and $\varepsilon$ are respectively the elementary charge and the permittivity of the liquid phase, $n_{j}$ and $z_{j}$ are respectively the concentration and the valence of ionic species $j$, and $\ell$ is a region index, $\ell=1$ for $a<r<b$, and $\ell=0$ for $b<r<c$. We assume that both $\varepsilon$ and $\rho_{\text {fix }}$ are constant.

The conservation of the $j$ th ionic species leads to

$\frac{\partial n_{j}}{\partial t}=D_{j}\left[\nabla^{2} n_{j}+\frac{z_{j} e}{k T}\left(\nabla n_{j} \cdot \nabla \phi+n_{j} \nabla^{2} \phi\right)\right]-\nabla n_{j} \cdot \mathbf{v}$

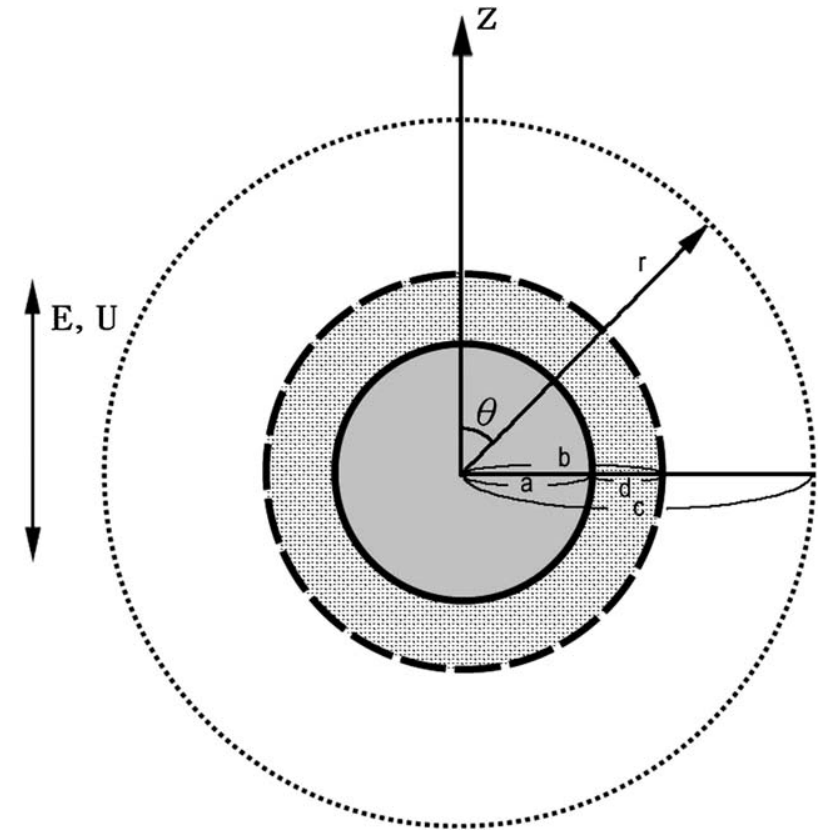

Fig. 1. A spherical dispersion of soft particles, where a particle comprises a rigid core of radius $a$ and a membrane layer of thickness $d$, is simulated by a unit cell model where a cell comprises a representative particle and a concentric spherical liquid shell of radius $c$. The spherical coordinates $(r, \theta, \varphi)$ are adopted with origin located at the center of the representative particle. $\mathbf{E}$ and $\mathbf{U}$ are respectively the applied electric field and the electrophoretic velocity of a particle.

where $D_{j}$ is the diffusivity of ionic species $j, k$ is the Boltzmann constant, $T$ is the absolute temperature, and $\mathbf{v}$ is the liquid velocity.

If we assume that the liquid phase is an incompressible Newtonian fluid with constant physical properties, then the flow field can be described by

$\nabla \cdot \mathbf{v}=0$

$\rho_{\mathrm{f}} \frac{\partial \mathbf{v}}{\partial t}-\nabla p+\eta \nabla^{2} \mathbf{v}-\rho \nabla \phi-\ell \gamma \mathbf{v}=0$,

where $p$ is the pressure, $\eta$ and $\rho_{\mathrm{f}}$ are respectively the viscosity and the density of the liquid phase, and $\gamma$ is a friction coefficient characterizing the flow of liquid in the membrane layer.

For convenience, $\phi, n_{j}, \mathbf{v}$, and $p$ are all decomposed into an equilibrium term and a perturbed term. That is,

$\phi(r, \theta, t)=\phi_{\mathrm{e}}(r)+\delta \phi(r, \theta) E_{z} e^{-i \omega t}$,

$n_{j}(r, \theta, t)=n_{j}^{\mathrm{e}}(r, \theta)+\delta n_{j}(r, \theta) E_{z} e^{-i \omega t}$,

$\mathbf{v}(r, \theta, t)=\mathbf{v}_{\mathrm{e}}+\delta \mathbf{v}(r, \theta) E_{z} e^{-i \omega t}$,

$p(r, \theta, t)=p_{\mathrm{e}}(r, \theta)+\delta p(r, \theta) E_{Z} e^{-i \omega t}$.

The subscript and superscript e in these expressions represents equilibrium properties, and $\delta$ denotes a perturbed property. Note that since the liquid phase remains stagnant in the absence of $\mathbf{E}$, we must have $\nabla p_{\mathrm{e}}=0$ and $\mathbf{v}_{\mathrm{e}}=\mathbf{0} ; p_{\mathrm{e}}$ and $\mathbf{v}_{\mathrm{e}}$ are respectively the equilibrium pressure and the equilibrium liquid velocity. Substituting Eqs. (5)-(8) into Eqs. (1)-(4) leads to two subproblems, one for the equilibrium system and the other for the perturbed system. 
In the absence of $\mathbf{E}$, the Boltzmann distribution is applicable to the distribution of mobile ions, and the equilibrium electrical potential $\phi_{\mathrm{e}}$ is described by

$\nabla^{2} \phi_{\mathrm{e}}=-\sum_{j=1}^{2} \frac{z_{j} \hat{e} n_{j 0}}{\varepsilon} \exp \left(-\frac{z_{j} \hat{e} \phi_{\mathrm{e}}}{k T}\right)-\ell \frac{\rho_{\mathrm{fix}}}{\varepsilon}$,

where $n_{j 0}$ is the bulk concentration of the $j$ th ionic species. The following boundary conditions are assumed for $\phi_{\mathrm{e}}$ :

$\phi_{\mathrm{e}}=\zeta_{a}, \quad r=a$,

$\left.\phi_{\mathrm{e}}\right|_{r=b^{-}}=\left.\phi_{\mathrm{e}}\right|_{r=b^{+}}, \quad r=b$

$\left.\frac{\partial \phi_{\mathrm{e}}}{\partial r}\right|_{r=b^{-}}=\left.\frac{\partial \phi_{\mathrm{e}}}{\partial r}\right|_{r=b^{+}}, \quad r=b$,

$\frac{\partial \phi_{\mathrm{e}}}{\partial r}=0, \quad r=c$.

These expressions imply that the surface of the rigid core of a particle $(r=a)$ remains at a constant potential, both the electrical potential and the electric field are continuous on the membrane layer-liquid interface $(r=b)$, and there is no net current across the surface of a cell $(r=c)$.

The movement of a particle as a response to the application of $\mathbf{E}$ leads to a distortion in the ionic cloud surrounding it. Similarly to the case of a static applied electric field [24], this can be modeled by

$n_{j}=n_{j 0} \exp \left(-\frac{z_{j} \hat{e}\left(\phi+g_{j} E_{Z} e^{-i \omega t}\right)}{k T}\right)$,

where $g_{j}$ is a potential characterizing the distortion of ionic cloud. Following the treatment of Lee et al. [10], the governing equation for the perturbed ionic concentration $\delta n_{j}$ can be recovered. We obtain

$$
\begin{aligned}
-i \omega \delta & n_{j} \\
= & -D_{j}\left(\nabla^{2}\left(\delta n_{j}\right)+\frac{z_{j} \hat{e}}{k T}\left(\nabla n_{j}^{\mathrm{e}} \cdot \nabla\left(\delta n_{j}\right)+\nabla\left(\delta n_{j}\right) \cdot \nabla \phi_{\mathrm{e}}\right.\right. \\
& +\nabla\left(\delta n_{j}\right) \cdot \nabla\left(\delta \phi E_{Z} e^{-i \omega t}\right)+n_{j}^{\mathrm{e}} \nabla^{2}(\delta \phi)+\left(\delta n_{j}\right) \nabla^{2} \phi_{\mathrm{e}} \\
& \left.\left.+\left(\delta n_{j}\right) \nabla^{2}\left(\delta \phi E_{Z} e^{-i \omega t}\right)\right)\right) \\
& +(\delta \mathbf{u}) \cdot \nabla\left(n_{j}^{\mathrm{e}}+\delta n_{j} E_{Z} e^{-i \omega t}\right) .
\end{aligned}
$$

Equations (6) and (14) yield

$$
\begin{aligned}
\delta n_{j} E_{Z} e^{-i \omega t}= & n_{j 0}\left(\exp \left(-\frac{z_{j} \hat{e}\left(\phi+g_{j} E_{Z} e^{-i \omega t}\right)}{k T}\right)\right. \\
& \left.-\exp \left(-\frac{z_{j} \hat{e} \phi_{\mathrm{e}}}{k T}\right)\right) .
\end{aligned}
$$

The governing equations for the perturbed flow field are

$$
\begin{aligned}
\nabla \cdot\left(\delta \mathbf{v} E_{Z} e^{-i \omega t}\right)= & 0, \\
-i \omega \rho_{\mathrm{f}} \delta \mathbf{v} E_{Z} e^{-i \omega t}= & -\nabla \delta p E_{Z} e^{-i \omega t}+\eta \nabla^{2} \delta \mathbf{v} E_{Z} e^{-i \omega t} \\
& -\rho_{\mathrm{c}} \nabla \phi-\ell \gamma \delta \mathbf{v} E_{Z} e^{-i \omega t} .
\end{aligned}
$$

Substituting Eqs. (9) and (14) into Eq. (5) and taking $\nabla^{2}$ on both sides of the resultant expression gives

$$
\begin{aligned}
\nabla^{2}\left(\delta \phi E_{Z} e^{-i \omega t}\right)= & \sum_{j=1}^{2} \frac{z_{j} \hat{e} n_{j 0}^{\mathrm{e}}}{\varepsilon}\left(\operatorname { e x p } \left(-\frac{z_{j} \hat{e}}{k T}\left(\phi_{\mathrm{e}}+\delta \phi E_{Z} e^{-i \omega t}\right.\right.\right. \\
& \left.\left.\left.+g_{j} E_{Z} e^{-i \omega t}\right)\right)-\exp \left(-\frac{z_{j} \hat{e}}{k T} \phi_{\mathrm{e}}\right)\right) .
\end{aligned}
$$

The following boundary conditions are assumed for $\delta \phi$ :

$\nabla\left(\delta \phi E_{Z} e^{-i \omega t}\right) \cdot \hat{\mathbf{r}}=0, \quad r=a$,

$\left.\delta \phi E_{z} e^{-i \omega t}\right|_{r=b^{-}}=\left.\delta \phi E_{z} e^{-i \omega t}\right|_{r=b^{+}}, \quad r=b$,

$\left.\frac{\partial \delta \phi E_{z} e^{-i \omega t}}{\partial r}\right|_{r=b^{-}}=\left.\frac{\partial \delta \phi E_{z} e^{-i \omega t}}{\partial r}\right|_{r=b^{+}}, \quad r=b$,

$\delta \phi E_{z} e^{-i \omega t}=-E_{z} e^{-i \omega t} c \cos \theta, \quad r=c$.

These expressions imply that the surface conductivity of the particle is negligible and the dielectric constant of the particle is much smaller than that of the liquid phase. Also, the boundary condition of Shilov and Zharkikh [9] is adopted.

The following boundary conditions are assumed for the concentration field:

$\left(f_{j}-n_{j} \mathbf{v}\right) \cdot \hat{\mathbf{r}}=0, \quad r=a$,

$\left.n_{j}\right|_{r=b^{-}}=\left.n_{j}\right|_{r=b^{+}}, \quad r=b$,

$\left.\mathbf{f}_{j}\right|_{r=b^{-}}=\left.\mathbf{f}_{j}\right|_{r=b^{+}}, \quad r=b$,

$n_{j}=n_{j 0}, \quad r=c$.

In these expressions, $\mathbf{f}_{j}$ is the concentration flux of the $j$ th ionic species, and $\hat{\mathbf{r}}$ is the unit vector in the $r$-direction. Here, we assume that the surface of the rigid core of a particle is impermeable to ions, both the concentration of ionic species and its flux are continuous on the membrane layer-liquid interface, and the concentration of ionic species reaches the corresponding bulk value on the cell surface.

Suppose that the surface of the rigid core of a particle is noslip and both the velocity of the liquid and its normal stresses are continuous on the membrane layer-liquid interface. For the tangential stress across that interface, the boundary condition of Ochoa-Tapia and Whitaker [17] is adopted; that is, it is discontinuous and a stress-jump coefficient $\beta$ is used to characterize the discontinuity in shear stress. Also, we assume that the vorticity of fluid velocity vanishes on the cell surface, and there is no net fluid flow across that surface. These lead to the following boundary conditions for the flow field:

$$
\begin{aligned}
& \delta v_{r} E_{Z} e^{-i w t}=\left(U_{\mathrm{R}}+i U_{\mathrm{I}}\right) E_{Z} e^{-i \omega t} \cos \theta, \quad r=a, \\
& \delta v_{\theta} E_{Z} e^{-i w t}=-\left(U_{\mathrm{R}}+i U_{\mathrm{I}}\right) E_{Z} e^{-i \omega t} \sin \theta, \quad r=a, \\
& \left.\delta v_{r}\right|_{r=b^{-}}=\left.\delta v_{r}\right|_{r=b^{+}},\left.\quad \delta v_{\theta}\right|_{r=b^{-}}=\left.\delta v_{\theta}\right|_{r=b^{+}}, \quad r=b, \\
& \left.\sigma_{r r}^{\mathrm{T}}\right|_{r=b^{-}}=\left.\sigma_{r r}^{\mathrm{T}}\right|_{r=b^{+}},\left.\quad \sigma_{r r}^{\mathrm{E}}\right|_{r=b^{-}}=\left.\sigma_{r r}^{\mathrm{E}}\right|_{r=b^{+}}, \quad r=b, \\
& \left.\sigma_{r \theta}^{\mathrm{H}}\right|_{r=b^{-}}+\beta \sqrt{\eta \gamma} \delta v_{\theta}=\left.\sigma_{r \theta}^{\mathrm{H}}\right|_{r=b^{+}}, \quad r=b, \\
& \nabla \times \delta \mathbf{v} E_{Z} e^{-i \omega t}=0 \quad \text { and } \delta v_{\mathrm{r}} E_{Z} e^{-i \omega t}=0, \quad r=c .
\end{aligned}
$$

In these expressions, $\sigma_{r \theta}^{\mathrm{H}}$ is the $r \theta$-component of the hydrodynamic stress tensor $\sigma^{\mathrm{H}}, \sigma_{r r}^{\mathrm{E}}$ is the $r r$-component of the Maxwell 
stress tensor $\sigma^{\mathrm{E}}=\varepsilon\left(\mathbf{E E}-\mathbf{E}^{2} \mathbf{I} / 2\right)$, and $\sigma_{r r}^{\mathrm{T}}$ is the $r r$-component of the total stress tensor $\sigma^{\mathrm{T}}=\sigma^{\mathrm{H}}+\sigma^{\mathrm{E}}$.

\section{Electrophoretic mobility}

For the present case the electrophoretic mobility of a particle $\mu$ can be expressed as

$\mu=\mu_{\mathrm{R}}+i \mu_{\mathrm{I}}=\frac{\mathbf{U}}{\mathbf{E}}$,

where $\mu_{\mathrm{R}}$ and $\mu_{\mathrm{I}}$ are respectively the real and the imaginary parts of $\mu$. The forces acting on a particle include the electric force $\mathbf{F}_{\mathrm{e}}$ and the hydrodynamic $\operatorname{drag} \mathbf{F}_{\mathrm{h}}$. Therefore,

$\mathbf{F}_{\mathrm{h}}+\mathbf{F}_{\mathrm{e}}=\frac{4}{3} \pi a^{3}\left(\rho_{\mathrm{p}}-\rho_{\mathrm{f}}\right) \frac{\mathrm{d} v}{\mathrm{~d} t}$,

where $\rho_{\mathrm{p}}$ is the averaged density of a particle. If we let $\sigma$ be the surface charge density of a particle, then the magnitude of $\mathbf{F}_{\mathrm{e}}$ in the $z$-direction, $\mathbf{F}_{\mathrm{e} z}$, can be calculated by

$\mathbf{F}_{\mathrm{e}}=\iint_{r=b} \boldsymbol{\sigma}^{\mathrm{E}} \cdot \mathbf{n} \mathrm{d} s$.

$F_{\mathrm{e} z}$ can be expressed as

$F_{\mathrm{e} z}=\mathbf{F}_{\mathrm{e}} \cdot \boldsymbol{\delta}_{z}=\iint_{r=b}\left(\boldsymbol{\sigma}^{\mathrm{E}} \cdot \mathbf{n}\right) \cdot \boldsymbol{\delta}_{z} \mathrm{~d} s$,

where $\delta_{z}$ is the unit vector in the $z$-direction. It can be shown that

$$
\begin{aligned}
F_{\mathrm{e} z}= & \frac{4}{3} \pi \varepsilon \zeta_{a}^{2}\left[r^{2}\left(\frac{\partial \phi_{\mathrm{e}}}{\partial r}\right)\left(\frac{\partial \delta \phi E_{Z} e^{-i \omega t}}{\partial r}\right)\right. \\
& \left.+2 r\left(\frac{\partial \phi_{\mathrm{e}}}{\partial r}\right) \delta \phi E_{Z} e^{-i \omega t}\right]_{r=b} \cdot
\end{aligned}
$$

The magnitude of $\mathbf{F}_{\mathrm{h}}$ in the $z$-direction, $F_{\mathrm{h}}$, can be evaluated by

$$
\begin{aligned}
F_{\mathrm{h}}= & -\pi \rho_{\mathrm{f}} \int_{0}^{\pi}\left[r^{2} \sin \theta \frac{\partial}{\partial t}\left(\frac{\partial\left(\psi E_{Z} e^{-i \omega t}\right)}{\partial r}\right)\right]_{r=b} \mathrm{~d} \theta \\
& +\eta \pi \int_{0}^{\pi}\left[r^{4} \sin ^{3} \frac{\partial}{\partial r}\left(\frac{E^{2} \psi E_{Z} e^{-i \omega t}}{r^{2} \sin ^{2} \theta}\right)\right]_{r=b} \mathrm{~d} \theta \\
& -\pi \int_{0}^{\pi}\left[r^{2} \sin ^{2} \theta \rho_{\mathrm{c}} \frac{\partial \phi}{\partial \theta}\right]_{r=b} \mathrm{~d} \theta,
\end{aligned}
$$

where $\psi$ is the stream function of the flow field. The first term on the right-hand side of this expression is the force arising from the change of the momentum of the liquid, the second term is the hydrodynamic drag, and the third term is the electric force arising from the flow of the electrolyte solution. Note that in terms of $\psi$, the $r$ - and the $\theta$-components of $\delta \mathbf{v}, \delta v_{r}$ and $\delta v_{\theta}$, can be expressed as $\delta v_{r}=-\left(1 / r^{2} \sin \theta\right)(\partial \psi / \partial \theta)$ and $\delta v_{\theta}=(1 / r \sin \theta)(\partial \psi / \partial r)$, respectively.

The electrophoretic mobility $\mu$ is characterized by its magnitude, $|\mu|=\left(\mu_{\mathrm{R}}^{2}+\mu_{\mathrm{I}}^{2}\right)^{1 / 2}$, and the associated phase angle, $\tan ^{-1}\left(\mu_{\mathrm{I}} / \mu_{\mathrm{R}}\right)$; that is,

$\mu=\sqrt{\mu_{\mathrm{R}}^{2}+\mu_{\mathrm{I}}^{2}} e^{i \Theta}$, where $\Theta=\tan ^{-1}\left(\mu_{\mathrm{I}} / \mu_{\mathrm{R}}\right)$. The phase angle measures the phase difference between the electrophoretic velocity of a particle and the applied electric field. A negative $\Theta$ implies a phase lead, that is, $\mathbf{U}$ leads $\mathbf{E}$, and a positive $\Theta$ implies a phase lag. Since $\mathbf{E}=E_{Z} e^{-i \omega t} \mathbf{e}_{z}$, the corresponding $\mathbf{U}$ can be expressed as $\mathbf{U}=$ $\sqrt{U_{\mathrm{R}}^{2}+U_{\mathrm{I}}^{2}} E_{Z} e^{-i \omega t+\Theta} \mathbf{e}_{z}$.

\section{Results and discussion}

The governing equations and the associated boundary conditions are solved by first expressing each perturbed term in Eqs. (5)-(8) as the product of a position-dependent function and a time-dependent function. Note that the latter has the form of a wave function and can be treated separately from the former. A pseudo-spectral method based on Chebyshev polynomials [26] is adopted for the resolution of ordinary differential equations. Numerical simulations are conducted to examine the general behavior of the phenomenon under consideration. In particular, the influences of the key parameters, including the basic properties of a particle, the frequency of the applied electric field, the stress-jump coefficient, and the concentration of particles, are discussed. For illustration, the following values are assumed: $\varepsilon_{r}=78.5, \eta=8.904 \times 10^{-3}$ poise $\rho_{\mathrm{f}}=0.99704 \mathrm{~g} / \mathrm{cm}^{3}, \rho_{\mathrm{p}}=1.05 \mathrm{~g} / \mathrm{cm}^{3}, z_{1}=1, z_{2}=$ $-1, T=298.15 \mathrm{~K}, D_{1}=1.962297 \times 10^{-5} \mathrm{~cm}^{2} / \mathrm{s}$, and $D_{2}=$ $2.037051 \times 10^{-5} \mathrm{~cm}^{2} / \mathrm{s}$. These yield $\omega a^{2} / D_{1}=454.88 \omega^{*}$ and $\omega a^{2} / D_{2}=472.21 \omega^{*}$, where $\omega^{*}=\rho_{\mathrm{f}} \omega a^{2} / \eta$ is the scaled frequency of the applied electric field. Without loss of generality, we assume that the rigid core of a particle is positively charged in the following discussions. Two general cases, $Q_{\mathrm{fix}}=0$ and $Q_{\text {fix }} \neq 0$, are considered, $Q_{\text {fix }}=\left(\rho_{\text {fix }} a^{2} / \varepsilon \zeta_{\mathrm{a}}\right)$ being the scaled density of the fix charge in the membrane layer of a particle.

\section{Case 1. $Q_{\mathrm{fix}}=0$}

Let us consider first the case where the membrane layer of a particle is free of fixed charge. The influence of the scaled surface potential on the rigid core of a particle $\phi_{r}$ and the stress-jump coefficient $\beta$ on the magnitude of the scaled mobility of a particle $\mu_{\mathrm{m}}^{*}$ and the corresponding phase angle $\Theta$ for the case when the thickness of double layer is comparable to the radius of the rigid core of the particle are illustrated in Fig. 2. If $\beta=0$, since the membrane layer is free of fixed charge, the general behavior of $\mu_{\mathrm{m}}^{*}$ and $\Theta$ shown in this figure is similar to that for the case of rigid spherical dispersion [25]: (a) If $\phi_{r}$ is low, $\mu_{\mathrm{m}}^{*}$ decreases but $\Theta$ increases with the increase in the scaled frequency of the applied electric field $\omega^{*}$. (b) In general, $\mu_{\mathrm{m}}^{*}$ increases but $\Theta$ decreases with the increase in $\phi_{r}$. (c) If $\phi_{r}$ is sufficiently high, $\mu_{\mathrm{m}}^{*}$ has a local maximum and $\Theta$ has a negative (phase lead) local minimum. These observations can be explained by the effects of the inertia of a particle, double-layer deformation, and the acceleration period of an applied electric field [25]. Fig. 2 indicates that a positive $\beta$ has the effect of raising the value of $\mu_{\mathrm{m}}^{*}$, and the reverse is true if $\beta<0$. This is because a positive $\beta$ implies that the shear stress on the membrane-layer side of the membrane layer-liquid interface is smaller than that on the liquid side, 


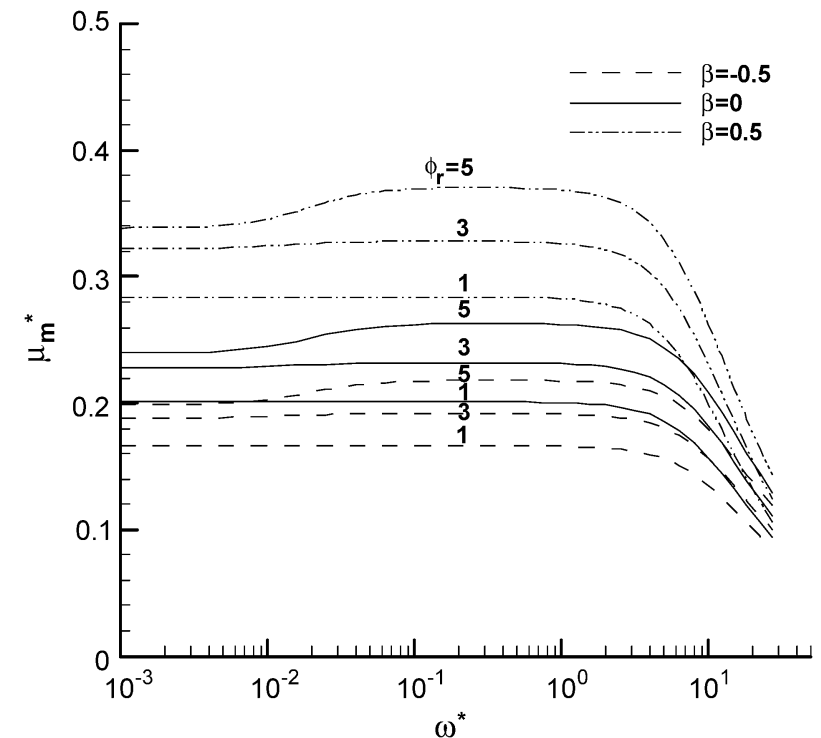

(a)

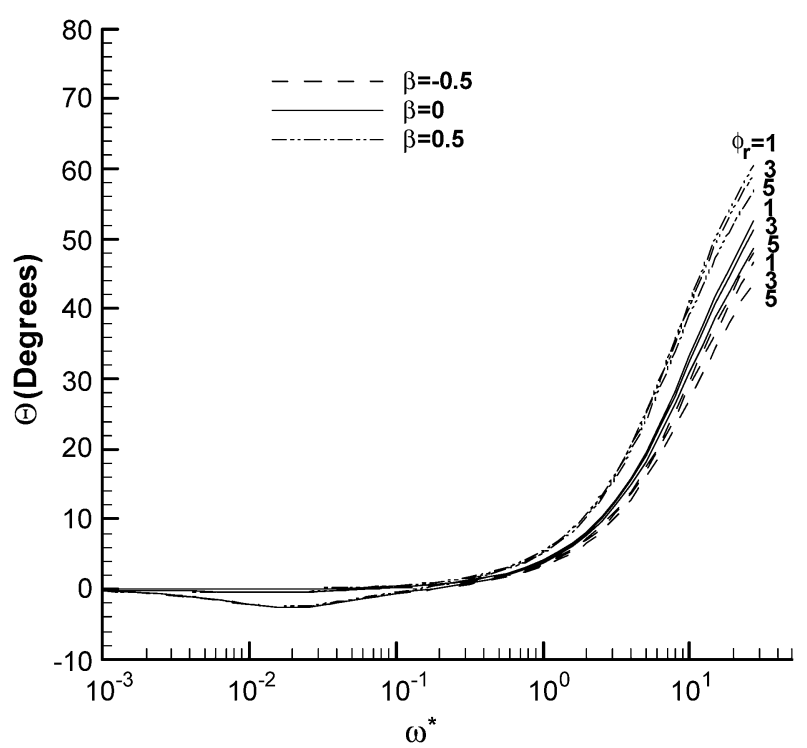

(b)

Fig. 2. Variation of scaled dynamic mobility $\mu_{\mathrm{m}}^{*}$ (a) and phase angle $\Theta$ (b) as a function of scaled frequency $\omega^{*}$ at various values of $\phi_{r}$ for the case where $\kappa a=1.0, \lambda a=5.0, Q_{\mathrm{fix}}=0.0, H=0.422$, and $d / a=0.5$.

and therefore, the drag acting on a particle decreases. The result for $\beta<0$ can be explained by similar reasoning. Note that for the same $|\beta|$, the degree of increase in $\mu_{\mathrm{m}}^{*}$ due to a positive $\beta$ is larger than the decrease in $\mu_{\mathrm{m}}^{*}$ arising from a negative $\beta$. This is because the friction of the membrane-layer of a particle has the effect of raising the shear stress for the flow of liquid inside. Therefore, if $\beta<0$, the higher shear stress on the membrane layer side of the membrane layer-liquid interface will be shielded by the friction of the membrane layer. Fig. 2 also reveals that a positive $\beta$ has the effect of raising the value of $\Theta$, and the reverse is true if $\beta<0$. This is because a positive $\beta$ leads to a larger $\mu_{\mathrm{m}}^{*}$, the momentum of a particle becomes greater accordingly, and, therefore, when the direction of the applied electric filed varies it takes longer for the particle to respond.

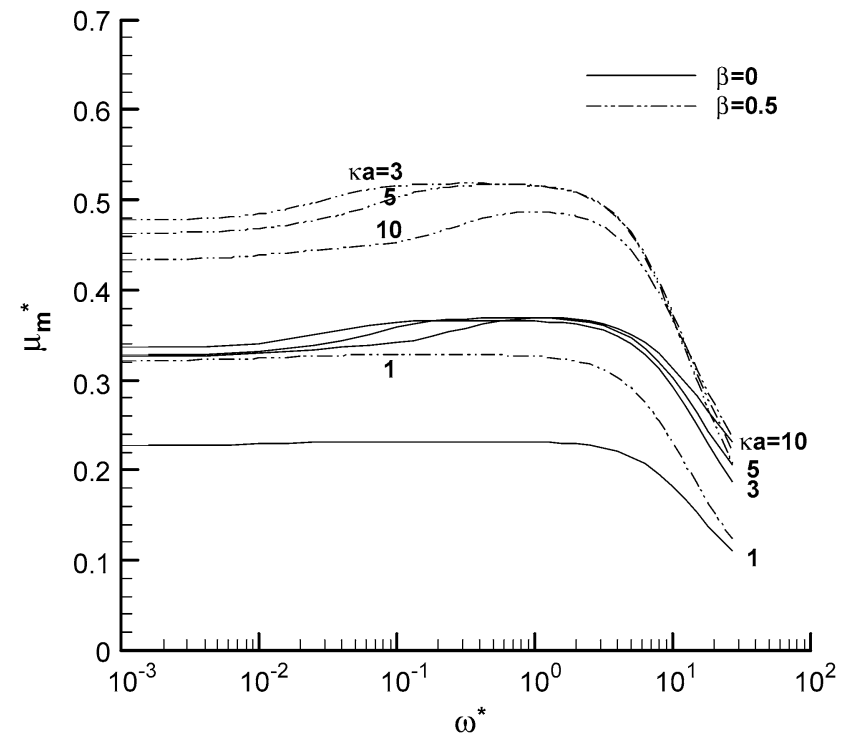

(a)

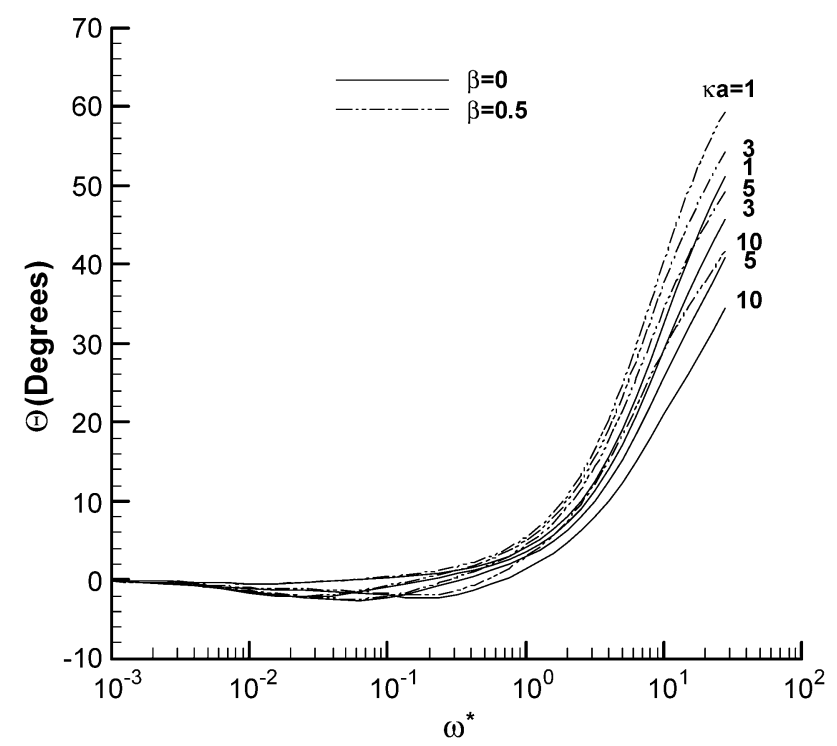

(b)

Fig. 3. Variation of scaled dynamic mobility $\mu_{\mathrm{m}}^{*}$ (a) and phase angle $\Theta$ (b) as a function of scaled frequency $\omega^{*}$ at various values of $\kappa a$ for the case where $\phi_{r}=3.0, \lambda a=5.0, Q_{\text {fix }}=0.0, H=0.422$, and $d / a=0.5$.

The influence of $\kappa a$ on the variation of the magnitude of the scaled mobility of a particle $\mu_{\mathrm{m}}^{*}$ and the corresponding phase angle $\Theta$ at two levels of $\beta$ is illustrated in Fig. 3. This figure reveals that $\mu_{\mathrm{m}}^{*}$ is a local maximum as $\omega^{*}$ varies, in general. This local maximum disappears, however, if $\kappa a$ is small, that is, if the double layer surrounding a particle is thick. The dependence of $\mu_{\mathrm{m}}^{*}$ as $\kappa a$ varies depends on the magnitude of $\omega^{*}$. As in the case of Fig. 2, a positive $\beta$ has the effect of raising the value of $\mu_{\mathrm{m}}^{*}$. Note that the smaller the value of $\kappa a$ the smaller the value of $\omega^{*}$ at which a phase lead occurs, and the larger the value of $\kappa a$ the more pronounced the degree of phase lead. These imply that the determination of the frequency at which the local maximum of $\mu_{\mathrm{m}}^{*}$ appears is not straightforward.

The influence of the concentration of particles on the electrophoretic behavior of a dispersion is shown in Fig. 4. This 


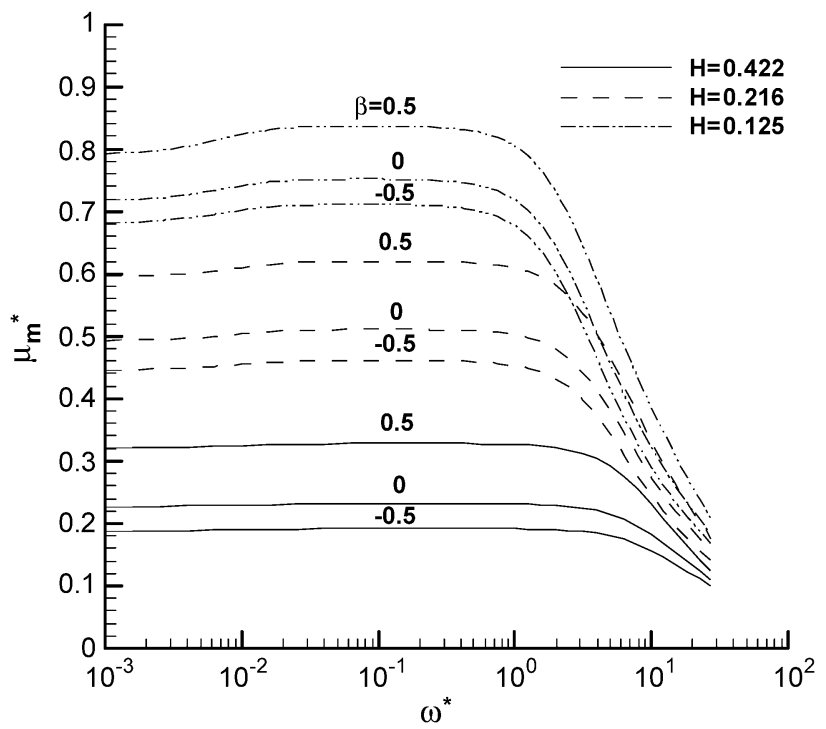

(a)

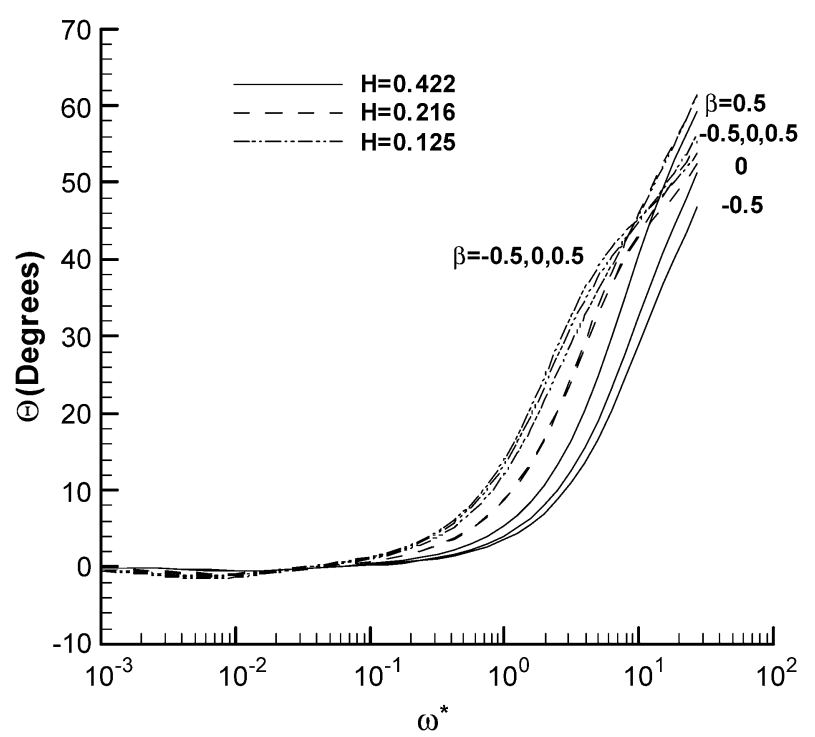

(b)

Fig. 4. Variation of scaled dynamic mobility $\mu_{\mathrm{m}}^{*}$ (a) and phase angle $\Theta$ (b) as a function of scaled frequency $\omega^{*}$ at various values of $H$ for the case where $\phi_{r}=3.0, \kappa a=1.0, \lambda a=5.0, Q_{\text {fix }}=0.0$, and $d / a=0.5$.

figure reveals that the mobility of a particle declines with the concentration of particles, a trend also observed in the case of a dispersion of rigid particles [10], and was explained by the effect of overlapping between neighboring double layers. Similarly to the cases of Figs. 2 and 3, $\mu_{\mathrm{m}}^{*}$ may have a local maximum and $\Theta$ have a negative local minimum as $\omega^{*}$ varies; both arise mainly from the effect of double-layer deformation. Note that if the concentration of particles is high, since the effect of double-layer deformation is confined, the phase lead in the mobility of a particle disappears. Fig. 4 indicates that if $\omega^{*}$ is high, the phase lag declines with the increase in $H$, which can also be explained by the effect of double-layer deformation being unimportant at a high $H$, making it easier for the ionic cloud to respond to variation in the applied electric field. As in the case of Fig. 2, a positive $\beta$ has the effect of raising the value of $\mu_{\mathrm{m}}^{*}$.

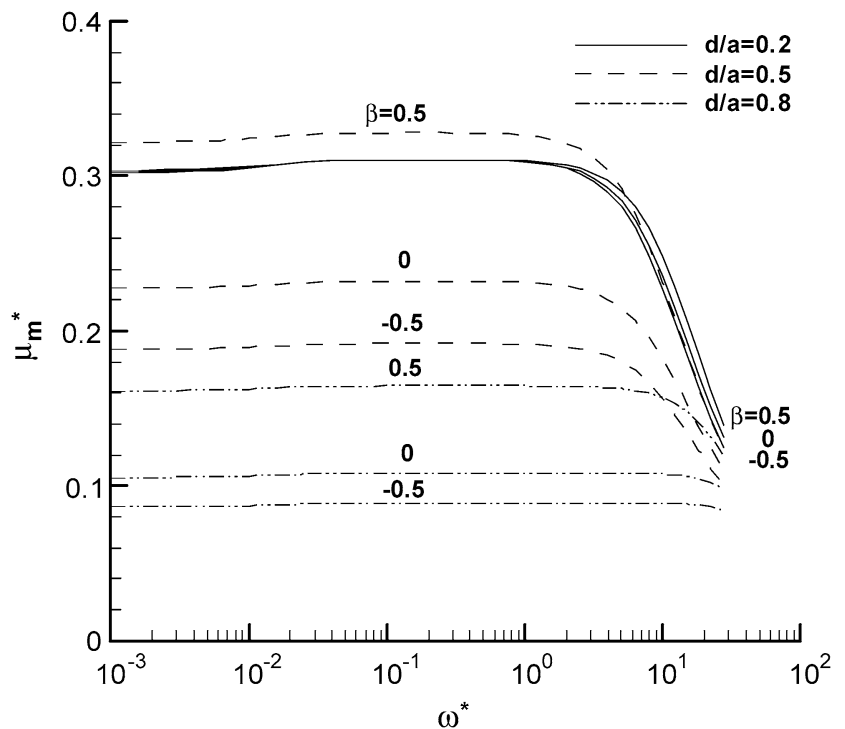

(a)

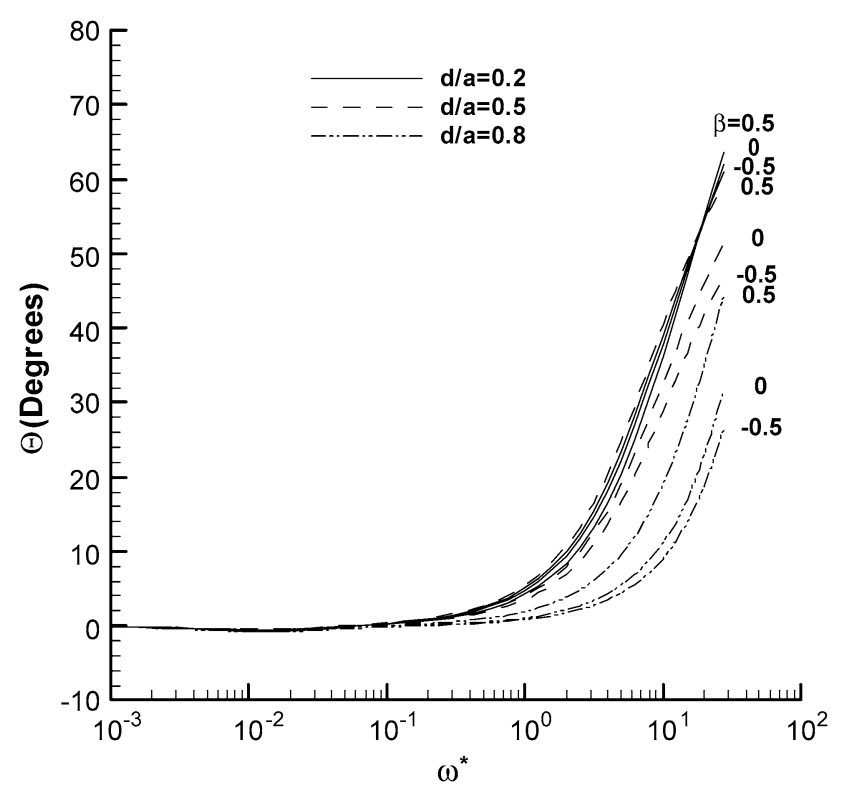

(b)

Fig. 5. Variation of scaled dynamic mobility $\mu_{\mathrm{m}}^{*}$ (a) and phase angle $\Theta$ (b) as a function of scaled frequency $\omega^{*}$ at various $(d / a)$ for the case where $\phi_{r}=3.0$, $\kappa a=1.0, \lambda a=5.0, Q_{\mathrm{fix}}=0.0$, and $c / a=2.0$.

Fig. 5 shows the influence of the relative thickness of the membrane layer of a particle $(d / a)$ on the electrophoretic behavior of a dispersion. In this case, since the membrane layer is free of fixed charge, its presence has the effect of raising the frictional resistance for fluid flow through it only, $\mu_{\mathrm{m}}^{*}$ decreases with the increase in $(d / a)$. Note that if $(d / a)$ is small, $\mu_{\mathrm{m}}^{*}$ is insensitive to the variation in $\beta$. This is because if the membrane layer is thin, the portion of a particle that can be influenced by the stress-jump condition is small.

Case 2. $Q_{\text {fix }} \neq 0$

Fig. 6 illustrates the variation of the scaled mobility of a particle $\mu_{\mathrm{m}}^{*}$ and the corresponding phase angle $\Theta$ as a function of the scaled frequency $\omega^{*}$ at three levels of $Q_{\text {fix }}$ for the case when 


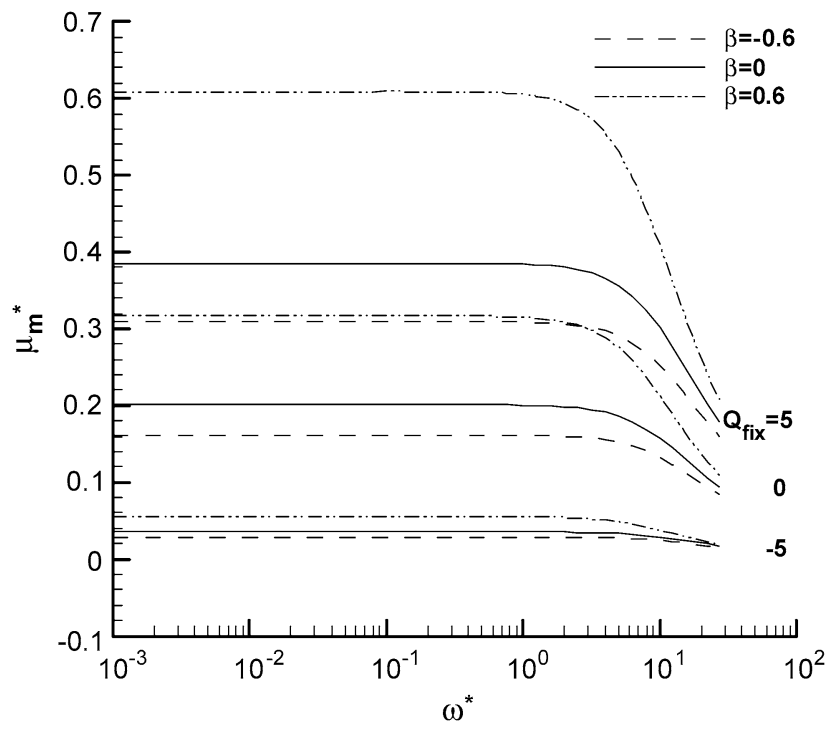

(a)

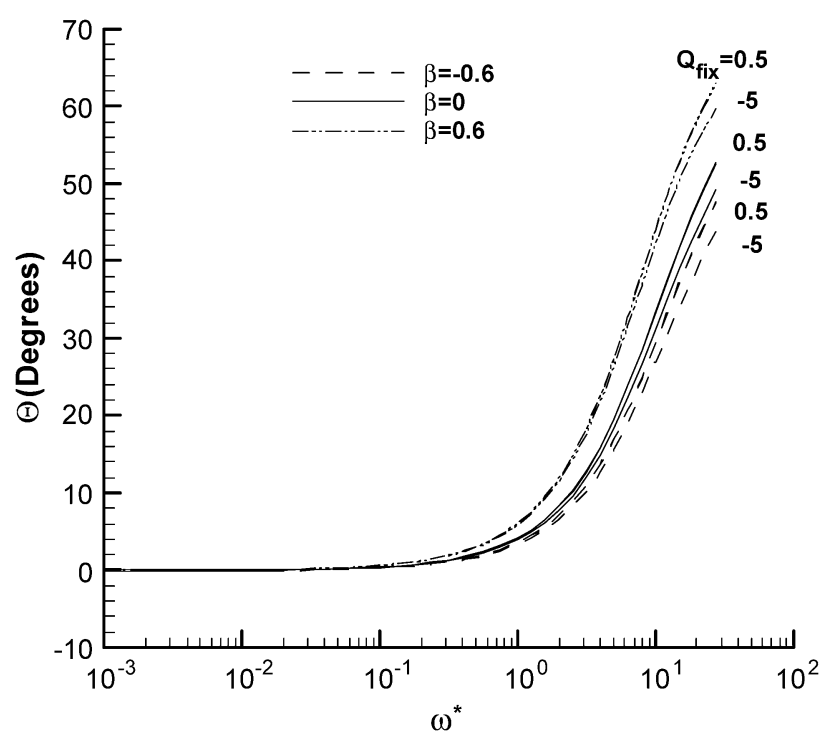

(b)

Fig. 6. Variation of scaled dynamic mobility $\mu_{\mathrm{m}}^{*}$ (a) and phase angle $\Theta$ (b) as a function of scaled frequency $\omega^{*}$ at various values of $\kappa a$ for the case when $\phi_{r}=1.0, \lambda a=5.0, \kappa a=1, H=0.422$, and $d / a=0.5$.

the rigid core of a particle is positively charged. This figure reveals that the magnitude of $\mu_{\mathrm{m}}^{*}$ follows the order $\mu_{\mathrm{m}}^{*}$ (positively charged membrane layer) $>\mu_{\mathrm{m}}^{*}$ (charge-free membrane layer) $>\mu_{\mathrm{m}}^{*}$ (negatively charged membrane layer), which is expected. Note that the effect of stress jump on $\mu_{\mathrm{m}}^{*}$ is more pronounced for the case when the membrane layer is positively charged than for that when it is negatively charged. This is because as the mobility of a particle increases due to a positively charged membrane layer, the drag on the particle increases also, and the influence of the discontinuity in the shear stress across the membrane layer-liquid interface increases accordingly. Note that the value of $\Theta$ for a positively charged membrane layer is about the same as that for a charge-free membrane layer and is larger than that for a negatively charged membrane layer.

Fig. 7 illustrates the variation of the scaled mobility of a particle $\mu_{\mathrm{m}}^{*}$ and the corresponding phase angle $\Theta$ as a function of

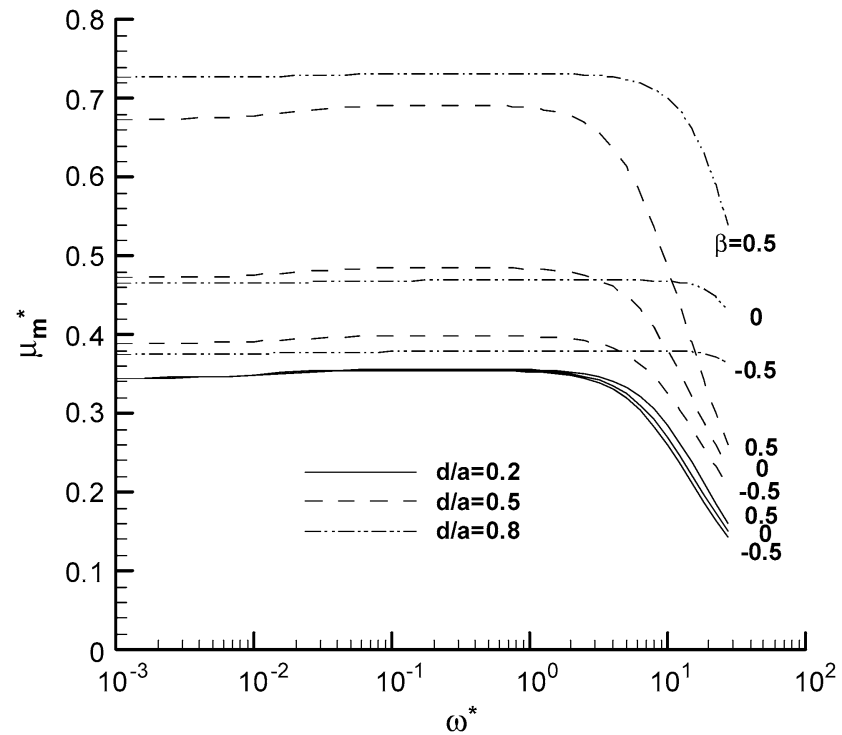

(a)

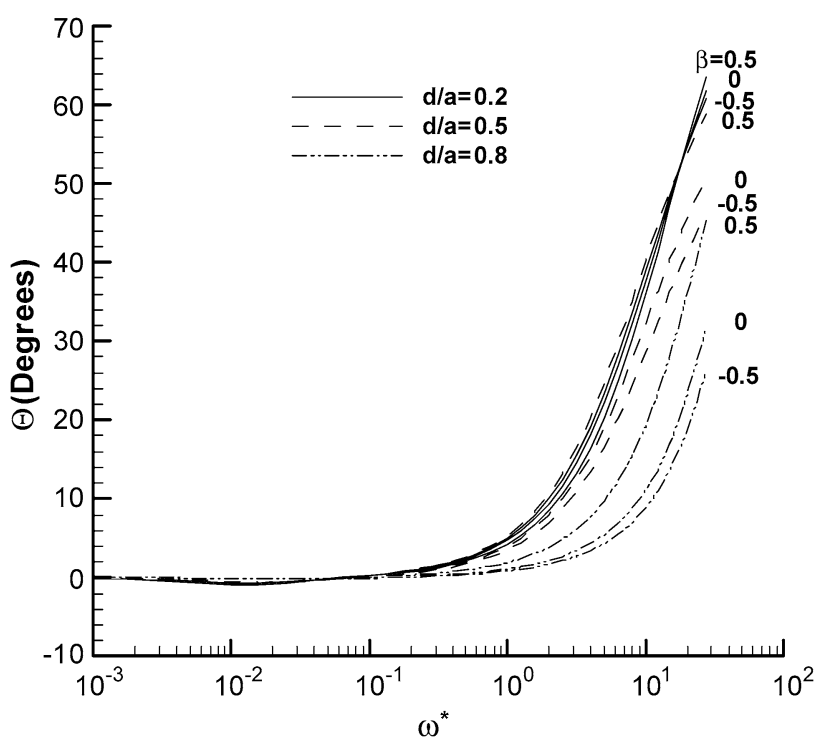

(b)

Fig. 7. Variation of scaled dynamic mobility $\mu_{\mathrm{m}}^{*}$ (a) and phase angle $\Theta$ (b) as a function of scaled frequency $\omega^{*}$ at various values of $(d / a)$ for the case when $\phi_{r}=3.0, \kappa a=1.0, \lambda a=5.0, Q_{\text {fix }}=5.0$, and $c / a=2.0$.

the scaled frequency $\omega^{*}$ at various values of $(d / a)$ for the case $Q_{\text {fix }}$ is positive. This figure indicates that the thicker the membrane layer of a particle, the larger the magnitude of its mobility is. As the thickness of the membrane layer increases, both the friction of liquid flow inside and the total fixed charge increase. The former is disadvantageous to the movement of a particle, but the latter is advantageous. The trends observed in Fig. 7 are the net results of those two factors.

\section{Conclusions}

The dynamic electrophoretic mobility of a spherical dispersion of soft particles, where a particle comprises a rigid core and a membrane layer, is analyzed for the case where the shear stress across the membrane layer-liquid interface is discon- 
tinuous, the so-called stress-jump condition. We conclude the following: (a) The higher the concentration of particles, due to a higher steric hindrance, the smaller the magnitude of the dynamic mobility of a particle. (b) If the effect of double-layer deformation is important, the magnitude of the mobility of a particle has a local maximum and its phase angle has a negative (phase lead) local minimum at a low to medium frequency of the applied electric field. This effect becomes unimportant when the frequency of the applied electric field is sufficiently high. (c) The stress-jump condition can have a significant influence on the mobility of a particle. The magnitude of the mobility of a particle with a positive stress-jump coefficient is larger than that of the corresponding particle when the stressjump coefficient is neglected, and the reverse is true when it is negative. (d) The effect of the stress-jump condition is influenced by the nature of the membrane layer of a particle. If the membrane layer is thin, the drag on a particle is small, and the effect of the stress-jump condition becomes inappreciable. (e) The effect of the stress-jump condition is pronounced if the sign of the fixed charge in the membrane layer of a particle is the same as that of the charge on the surface of its rigid core; the reverse is true if the fixed charge has a sign opposite to that of the charge on the surface of the rigid core.

\section{Acknowledgment}

This work is supported by the National Science Council of the Republic of China.

\section{References}

[1] R.J. Hunter, Colloids Surf. A 141 (1998) 37.

[2] R.W. O’Brien, J. Fluid Mech. 190 (1988) 71.

[3] R.P. Sawatzky, A.J. Babchin, J. Fluid Mech. 246 (1993) 321.

[4] C.S. Mangelsdorf, L.R. White, J. Chem. Soc. Faraday Trans. 88 (1992) 3567.

[5] H. Ohshima, J. Colloid Interface Sci. 179 (1996) 431.

[6] S. Levine, G.H. Neale, J. Colloid Interface Sci. 47 (1974) 520.

[7] H. Ohshima, Colloids Surf. A 159 (1999) 293.

[8] A.S. Dukhin, V. Shilov, Y. Borkovskaya, Langmuir 15 (1999) 3452.

[9] V.N. Shilov, N.I. Zharkikh, Y.B. Borkovskaya, Colloid J. 43 (1981) 434.

[10] E. Lee, F.Y. Yen, J.P. Hsu, J. Phys. Chem. B 105 (2001) 7239.

[11] E. Donath, V. Pastushenko, Bioelectrochem. Bioenerg. 6 (1979) 543.

[12] R.W. Wunderlich, J. Colloid Interface Sci. 88 (1982) 385.

[13] S. Levine, M. Levine, K.A. Sharp, D.E. Brooks, Biophys. J. 42 (1983) 127.

[14] K.A. Sharp, D.E. Brooks, Biophys. J. 47 (1985) 563.

[15] H. Ohshima, J. Colloid Interface Sci. 233 (2001) 142.

[16] J.J. Lopez-Garcia, C. Grosse, J. Horno, J. Colloid Interface Sci. 265 (2003) 341.

[17] J.A. Ochoa-Tapia, S. Whitaker, Int. J. Heat Mass Transfer 38 (1995) 2635.

[18] J.A. Ochoa-Tapia, S. Whitaker, Int. J. Heat Mass Transfer 38 (1995) 2647.

[19] G.S. Beavers, D.D. Joseph, J. Fluid Mech. 30 (1967) 197.

[20] A.V. Kuznetsov, Int. J. Heat Mass Transfer 24 (1997) 401.

[21] G.P.R. Sekhar, O. Sano, Phys. Fluids 15 (2003) 554.

[22] A. Bhattacharyya, G.P.R. Sekhar, Chem. Eng. Sci. 59 (2004) 4481.

[23] S. Kuwabara, J. Phys. Soc. Jpn. 14 (1959) 527.

[24] E. Lee, K.T. Chou, J.P. Hsu, J. Colloid Interface Sci. 280 (2004) 518.

[25] J.P. Hsu, E. Lee, F.Y. Yen, J. Phys. Chem. B 106 (2002) 4789.

[26] J.W. Chu, W.H. Lin, E. Lee, J.P. Hsu, Langmuir 17 (2001) 6289. 